\title{
Potential PDF sensitivity at LHCb
}

\author{
Ronan McNulty on behalf of the LHCb Collaboration * \\ School of Physics, University College Dublin, \\ Belfield, Dublin 4. Ireland.
}

\begin{abstract}
A review of the potential sensitivity of the $\mathrm{LHCb}$ experiment to the parton distribution functions is given. Studies of dimuon events coming from Z, W and low mass Drell Yan production are presented and compared to MSTW theoretical predictions.
\end{abstract}

\section{Introduction}

$\mathrm{LHCb}$ 2], one of the four main experiments at the LHC, is designed to make measurements of $\mathrm{CP}$ violation and rare $\mathrm{B}$ decays in the forward region $(1.9 \leq \eta \leq 4.9)$. However, it can also make precision electroweak measurements at high rapidities which enable it to probe the proton parton distribution functions (PDFs) in hitherto unmeasured regions.

Part of LHCb's pseudorapidity range $(1.9 \leq \eta \leq 2.5)$ overlaps that of ATLAS and CMS, however measurements made at $\eta>2.5$ are unique to LHCb. Other distinguishing features are the lower luminosity which reduces the number of pile-up events (one year of nominal running should collect $2 \mathrm{fb}^{-1}$ of data), and the ability to trigger on low transverse momentum muons, $p_{T}$, down to $1 \mathrm{GeV} / \mathrm{c}$.

We describe results of simulation studies [3] that show $\mathrm{LHCb}$ will be able to trigger on and reconstruct $\mathrm{Z}$ and $\mathrm{W}$ bosons with high efficiency and purity. Phenomenologically $\mathrm{LHCb}$ differs from the other experiments since the production of $\mathrm{W}$ and $\mathrm{Z}$ here occurs predominantly through sea-valence interactions, in contrast to the sea-sea interactions in the central region [4]. We also present studies of the Drell-Yan production of low-mass muon pairs which suggest that LHCb can isolate such events down to invariant masses, $Q^{2}$, of $5 \mathrm{GeV} / \mathrm{c}^{2}$.

We discuss the implications that such measurements will have on constraining the proton PDFs: in particular, the Drell-Yan final states allow us to probe values of the parton momentum fraction, $x$, down to $2 \times 10^{-6}$, a previously unexplored region.

\section{Measurements of $\mathrm{Z}$ production}

$\mathrm{Z}$ boson decays to dimuons possess a distinctive signature that allow them to be triggered [5] and reconstructed with high efficiency and purity. The dimuon trigger at LHCb is very loose and requires two muon candidates whose summed transverse momentum exceeds $1.6 \mathrm{GeV} / \mathrm{c}$. Offline selections suppress background processes by requiring: that both muons candidates have little activity in the hadronic calorimeter; that the higher and lower transverse momentum muons are greater than $20 \mathrm{GeV} / \mathrm{c}$ and $15 \mathrm{GeV} / \mathrm{c}$ respectively; that both have impact parameters consistent with the primary interaction; and their combined invariant mass lies within $20 \mathrm{GeV} / \mathrm{c}^{2}$ of the $\mathrm{Z}$ mass. Backgrounds have been studied [6] from other electroweak processes $(Z \rightarrow \tau \tau$ decays, $Z \rightarrow b b$ decays, $\mathrm{W}+$ jet production, WW production); quark production ( $b b, c c, t t$, and single top); and pions and kaons misidentified as muons.

${ }^{*}$ The author would like to acknowledge the support of Science Foundation Ireland 
For $\mathrm{Z}$ bosons decaying to two muons which are within the LHCb acceptance, the selection efficiency is $91 \pm 1 \%$ and the purity is estimated to be $97 \pm 3 \%$ with the dominant background $(3 \pm 3 \%)$ being mis-identified hadrons whose contribution will be precisely determined from data. After just $50 \mathrm{pb}^{-1}$ of data, the cross-section measurement will be limited by systematic uncertainties to about 1\%, assuming the machine luminosity can be precisely determined. The dominant uncertainty in the theoretical prediction comes from knowledge of the PDFs and is from 2-5\% depending on rapidity, and thus an experimental measurement of the $\mathrm{Z}$ cross-section will constrain the PDFs.

The sensitivity of the PDFs to a measurement of the $\mathrm{Z}$ cross-section can be seen in Figure 1] where we have plotted the variation in the differential cross-section produced using the MCFM generator [7] with $\pm 1 \sigma$ variations in each of the 20 eigenvectors [8] in the MSTW07 parametrisation 9]. Superimposed upon these are the expected experimental uncertainties with $1 \mathrm{fb}^{-1}$ of data, assuming that the luminosity is known.

Current estimates for the precision on a luminosity determination are from 1 to $3 \%$ using the beam-gas technique [10], or by measuring the elastic two-photon production of leptons [11. However, even in the absence of a precise luminosity determination, information about the PDFs can be derived from the shape of the differential distribution $\frac{d \sigma}{d y}$; only the overall normalisation is fixed by the luminosity.

\section{$3 \mathrm{~W}$ production}

W boson decays to a lepton
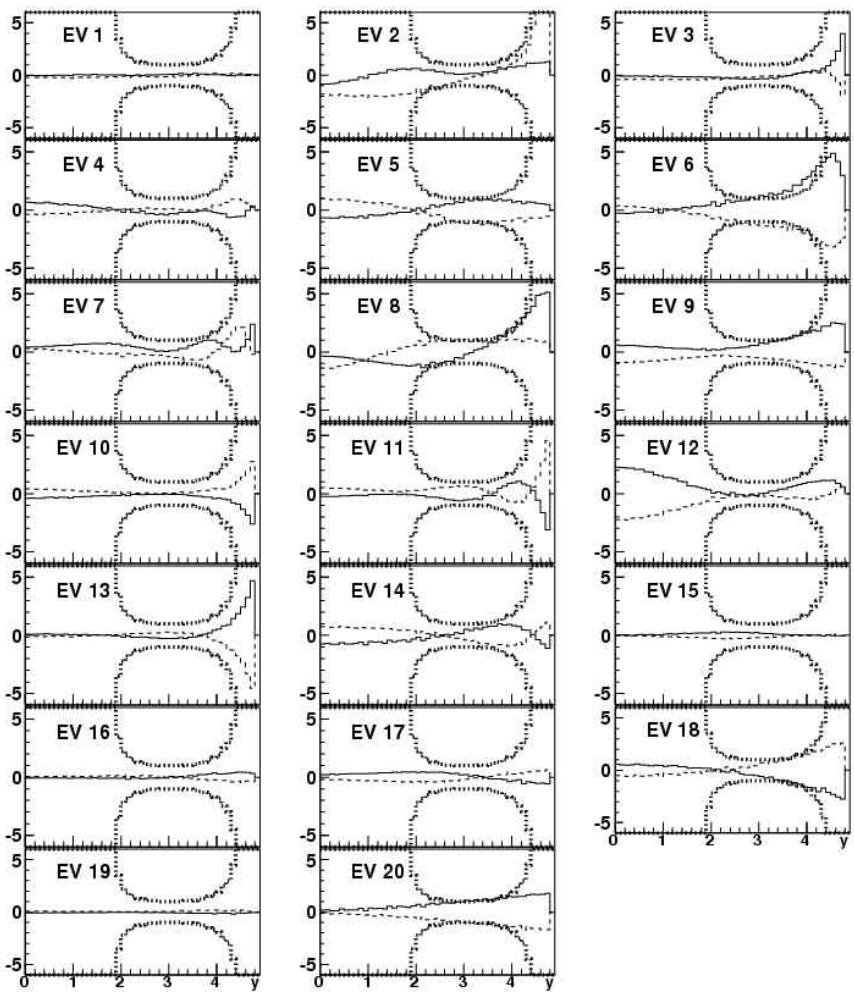

Figure 1: A comparison of the theoretical and experimental precision on the differential cross-section for $\mathrm{Z}$ boson production as a function of rapidity, $y$, using $1 \mathrm{fb}^{-1}$ of $\mathrm{LHCb}$ data for each of the 20 eigenvectors in the MSTW07 parametrisation. The $+1(-1) \sigma$ uncertainties are indicated with a continuous (dashed) line. The experimental uncertainty is indicated with a dotted line.

plus neutrino have previously been observed at hadron colliders by observing high momentum leptons in association with missing transverse energy, a quantity that can not be measured well at $\mathrm{LHCb}$ since it is only instrumented in the for- 
ward region. Nonetheless, our studies indicate that the presence of a single high transverse momentum muon which makes up the dominant observed activity in the event, is sufficent to select a rather clean samples of Ws.

First we require the presence of a high transverse momentum muon, $p_{T}>30 \mathrm{GeV} / \mathrm{c}$. Defining the asymmetry, $A$, between two quantities $x$ and $y$ to be $A(x, y)=\frac{x-y}{x+y}$ we compare the muon momentum, $p^{\mu}$, to the summed momentum of every other charged particle detected in $\mathrm{LHCb}, p^{\text {rest }}$, and require that $A\left(p^{\mu}, p^{\text {rest }}\right)>0.85$. Whereas the signal is peaked towards 1 , backgrounds coming from semi-leptonic $b$ decays or mis-identified pions are strongly peaked towards -1 . The combination of these requirements has an overall selection efficiency of about $33 \%$ and a purity of $83 \%$. Again, the dominant background is due to misidentifying a pion as a muon. It is noteworthy that in LHCb, Z decays form a small $(3 \%)$ but irreducible background, caused when only one of the dimuons enters the LHCb acceptance.

A full study of systematic errors is ongoing though it is expected that a similar experimental precision to the $\mathrm{Z}$ channel (1\%) can be obtained. The comparison to the theoretical prediction is complicated by the fact that the $\mathrm{W}$ boson itself can not be reconstructed. Consequently we have compared our distributions to the theoretical expectations for the differential distributions expressed as a function of lepton rapidities. Once again knowledge of the PDFs are the dominant uncertainties (ranging from $2 \%$ to $4 \%$ for MSTW07) and so the experimental data can constrain the PDFs.

The uncertainty due to luminosity can be removed by comparing ratios and asymmetries of the $W^{+}, W^{-}$and $Z$ differential cross-sections in such a way as to minimise or maximise the dependence on the PDFs [12]. The ratio $R_{Z W}=\frac{\sigma_{Z}}{\sigma_{W+}+\sigma_{W-}}$ is then almost independent of PDF uncertainties and thus constitutes a precise electroweak prediction for the LHC. The ratio $R_{ \pm}=\frac{\sigma_{W+}}{\sigma_{W-}}$ has an uncertainty that rises from $<1 \%$ at $y=0$ to $20 \%$ at $y=4.5$ and at high rapidities, which only LHCb can measure, is sensitive to the large- $x u / d$ ratio of partons. Measuring the asymmetry $A(W+, W-)$ will greatly constrain the PDFs since the current theoretical uncertainty on this quantity is about $10 \%$ at all rapidities.

\section{Low invariant mass Drell-Yan production}

Dimuons produced below the $\mathrm{Z}$ resonance can be recorded down to an invariant mass of $1.6 \mathrm{Gev} / \mathrm{c}^{2}$ using the dimuon trigger. However, isolating the signal from the orderof-magnitude larger backgrounds coming from semileptonic heavy quark decays and pion misidentification is challenging. Considering the topology of the signal leads us to consider five observables: the momenta of each of the muons $\left(p^{\mu 1}, p^{\mu 2}\right)$; the summed momenta of all particles found within a cone of one unit of phi and pseudorapidity about each muon $\left(p^{\text {cone } 1}, p^{\text {cone } 2}\right)$; and the summed momenta of any other activity in the event $\left(p^{\text {rest }}\right)$. Five asymmetries are defined: $A\left(p^{\mu 1}, p^{\mu 2}\right) ; A\left(p^{\mu 1}, p^{\text {cone } 1}\right) ; A\left(p^{\mu 2}, p^{\text {cone } 2}\right) ; A\left(p^{\mu 1}+p^{\mu 2}, p^{\text {rest }}\right)$; $A\left(p^{\text {cone } 1}+p^{\text {cone } 2}, p^{\text {rest }}\right)$; whose shapes are almost independent of the invariant mass of the muons. These variables are combined to form a likelihood. Cutting progressively harder on this likelihood increases the purity of the signal though at the expense of efficiency. Our studies show that above a dimuon invariant mass of $10 \mathrm{GeV} / \mathrm{c}^{2}$, purities above $95 \%$ can be obtained retaining between $15 \%$ and $50 \%$ of triggered signal events, so that the total number of selected events per mass bin is roughly constant. Below $10 \mathrm{GeV} / \mathrm{c}^{2}$ pure signals can not be obtained although measurements of the cross-section may be possible down to $5 \mathrm{GeV} / \mathrm{c}^{2}$ where $5 \%$ of the signal events can be retained with a purity of $70 \%$. 
The experimental precision obtainable on the double differential distribution, $d^{2} \sigma / d Q^{2} d y$, is compared with the current theoretical uncertainties due to the PDFs in Figure 2. With $1 \mathrm{fb}^{-1}$ of data, and with bins of 0.1 in rapidity and $2 \mathrm{GeV} / \mathrm{c}^{2}$ in mass, a statistical precision of a few percent per bin is obtainable, though systematic uncertainties remain to be determined and could be larger at low masses where the backgrounds are greater. The theoretical uncertainties at low invariant masses is substantial and driven by knowledge of the small- $x$ gluon, approaching $60 \%$ at high rapidities for $Q^{2}=8 \mathrm{GeV} / \mathrm{c}^{2}$.

Within the first year of data-taking, LHCb should thus start to constrain the gluon PDF and make significant improvements on current knowledge after a few years. At high rapidities and values of $Q^{2}=5 \mathrm{GeV} / \mathrm{c}^{2}$, LHCb will be able to probe $x$ values down to $2 \times 10^{-6}$ where the PDFs are currently unknown.

\section{Conclusions}

The $\mathrm{LHCb}$ experiment can access a unique range of $x$ and $\mathrm{Q}^{2}$ at the LHC. Ratios and asymmetries of the $\mathrm{W}$ and $\mathrm{Z}$ cross-sections will be experimentally determined to $1 \%$ constraining the PDFs which currently have a theoretical uncertainty of $2-5 \%$ at $Q^{2} \approx 90 \mathrm{GeV} / \mathrm{c}^{2}$. Studies of low invariant mass DrellYan dimuon states indicate that $\mathrm{LHCb}$ can rapidly improve the knowledge of PDFs down to $x$ values of $2 \times 10^{-6}$ and $\mathrm{Q}^{2}$ of $5 \mathrm{GeV} / \mathrm{c}^{2}$ and significantly improve our knowledge of the small- $x$ gluon.

\section{References}

[1] Slides: http://indico. cern. $\mathrm{ch} /$ getFile.py/access? contribId=314\&sessionId=17\&res Id=0\&material Id=slides\&conf Id=24657

[2] LHCb Collaboration, Reoptimised Detector Design and Performance TDR, CERN-LHCC-2003-030

[3] LHCb Computing TDR CERN-LHCC-2005-019.

[4] A.D. Martin et al., Parton Distributions and the LHC: $W$ and Z Production, hep-ph/9907231

[5] LHCb Trigger System TDR, CERN-LHCC-2003-031.

[6] T.Sjostrand, S.Mrenna, P.Skands, Pythia 6.4 physics and manual JHEP 0605:026 (2006).

[7] M. Campbell, R.K. Ellis, Phys. Rev. D62:114012 (2000).

[8] A.D.Martin et al., Uncertainties of predictions from parton distributions. hep-ph/0211080

[9] R. Thorne, Parton Distributions for the LHC, DIS2007 Munich, Proceedings doi:10.3360/dis.2007.27

[10] M. Ferro-Luzzi, Proposal for an absolute luminosity determination, Nucl. Inst. Meth. A553 (2005) 388.

[11] A.G. Shamov, V.I. Telnov, Nucl. Inst. Meth. A494 (2002) 51. See also LHCb note LHCb-2008-001.

[12] A. Tricoli et al., Uncertainties on $W$ and $Z$ production at the LHC Proc. HERA-LHC hep-ex/0509002

[13] J. Stirling, Plenary talk at this conference: http://indico. cern. $\mathrm{ch} /$ materialDisplay $\cdot$ py? contribId=18\&sessionId=5\&material Id=slides\&conf Id=24657 